\title{
HLA Multi Agent/Short-Lived Ontology Platform for Enterprise Interoperability
}

\author{
Gregory Zacharewicz ${ }^{1}$, Olivier Labarthe ${ }^{2}$, David Chen ${ }^{1}$, and Bruno Vallespir ${ }^{1}$ \\ ${ }^{1}$ LAPS / GRAI, Université de Bordeaux - CNRS, 351, Cours de la Libération, \\ 33405 TALENCE Cedex, France \\ ${ }^{2}$ CIRRELT, Université Laval, 2325 rue de la Terrasse, bureau 2535, \\ Québec City, Québec, Canada \\ \{gregory.zacharewicz, david.chen, \\ bruno.vallespir\}@ims-bordeaux.fr, olivier.labarthe@cirrelt.ca
}

\begin{abstract}
This paper aims at proposing an specification of the Federation oriented Enterprise Interoperability concept, using Multi Agent / HLA paradigm and the rising notion of Short-Lived Ontology. We give first, a review of Enterprise Interoperability. Then, we recall on Artificial Agent Concept and HLA Standard that appear to be adequate to support execution of the studied concept. Indeed, on the one hand Agent dialogue fits the concept of information exchange in a federated enterprise interoperability approach, on the other hand the HLA standard, initially designed for military M\&S purpose, can be transposed for enterprise interoperability at the implementation level, reusing the years of experiences in distributed systems. From these postulates, we propose the first Agent/HLA Short-Lived Ontology framework for distributed enterprise interoperability solution in the federated enterprise approach.
\end{abstract}

Keywords: Enterprise Interoperability, Multi Agent Systems, Distributed Simulation, HLA, Ontology.

\section{Introduction}

In the globalised economic context, the competitiveness of an enterprise depends not only on its internal productivity and performance, but also on its skill to collaborate with others. This necessity led to the development of a new concept called interoperability that allows improving collaborations between enterprises. No doubt, in such context where more and more networked enterprises are developed; enterprise interoperability is seen as a more suitable solution to total enterprise integration. Since the beginning of 2000, several European research projects have been launched to develop enterprise interoperability (e.g. INTEROP [1]). Three main research themes or domains that address interoperability issues were identified, namely: (1) Enterprise Modeling (EM) dealing with the representation of the internetworked organization to establish interoperability requirements; (2) Architecture \& Platform (A\&P) defining the implementation solution to achieve interoperability; (3) Ontologies (ON) addressing semantics necessary to assure interoperability. 
This paper proposes a new contribution of Information Technology (IT) architecture and platform to implement Enterprise Interoperability. In the first part, we present the various approaches of interoperability and the current consideration of interoperability stated as conclusion of the Interop Network of Excellence (FP6, 508011). Then, we recall the concepts of software Agent and the High Level Architecture (HLA) [2], i.e. a standard for distributed simulation.

Next, from our experience, we propose to investigate three aspects of interoperability: time management, Enterprise Ontologies and the privacy of data. Indeed. Computer science Ontologies, Artificial Agent language, and Object / Interaction in HLA can give keys to two first considerations. As well, the experience coming from Information Systems (IS) and M\&S programming can be studied to keep data safe to address third point. From these postulates, we specify a plat-form implementation using HLA and Software Agents' autonomous dialogue concepts, to the concern of distributed federated Enterprise Interoperability models.

\section{Basic Concept of Interoperability}

Enterprise Interoperability refers to the ability of interactions between enterprise systems. The interoperability is considered as significant if the interactions can take place at least at the three different levels: data, services and process, with a semantics defined in a given business context. Establishing interoperability means to relate two systems together and remove incompatibilities. Our goal is to tackle interoperability problems through the identification and passing barriers which prevent interoperability to happen. The interoperability has been historically established as:

Integrated approach. there exists a common format for all models. This format must be as detail as models. The common format is not necessarily a standard but must be agreed by all parties to elaborate models and build systems.

Unified approach. there exists a common format but only at a meta-level. This metamodel is not an executable entity as it is in the integrated approach but provides a mean for semantic equivalence to allow mapping between models.

Federated approach. there is no common format. To establish interoperability, parties must accommodate on the fly. Using federated approach implies that no partner imposes their models, languages and methods of work.

Today, most of the approaches developed are unified ones. Using the federated approach to develop Enterprise Interoperability is most challenging and few activities have been performed in this direction. The federated approach aims to develop full interoperability and is particularly suitable for an inter-organizational environment (such as networked enterprises, virtual enterprises, etc.).

\section{HLA Recalls}

The High Level Architecture (HLA) is a software architecture specification that defines how to create a global execution composed of distributed simulations and 
software. This standard was originally introduced by the Defense Modeling and Simulation Office (DMSO) of the US Department Of Defense (DOD). The original goal was reuse and interoperability of military applications, simulations and sensors.

In HLA, every participating application is called federate. A federate interacts with other federates within a HLA federation, which is in fact a group of federates. The HLA set of definitions brought about the creation of the standard 1.3 in 1996, which evolved to HLA 1516 in 2000 [2] The interface specification of HLA describes how to communicate within the federation through the implementation of HLA specification: the Run Time Infrastructure (RTI). In order to respect the temporal causality relations in the execution of distributed computerized applications; HLA proposes to use classical synchronization mechanisms [2].

An HLA federation is composed of federates and a Run Time Infrastructure (RTI).A federate is a HLA compliant program, the code of that federate keeps its original features but must be extended by other functions to communicate with other members of the federation. The RTI supplies services required by distributed executions, it routes messages exchanged between federates. A federate can, through the services proposed by the RTI, "Publish" and "Subscribe" to a class of shared data.

\section{Agent-Based Distributed Simulations}

The Multi-Agents System (MAS) [3] concentrates on the study of the collective behavior which results from the organization and interactions of agents for the resolution of problems. A MAS is a distributed system in which there is generally no centralized control or global point of view. A MAS is composed of agents which act in an autonomous way but do not locally have the knowledge, the resources or the information required to ensure the coherence of the concerted actions in a MAS. This section is dedicated to the presentation of the agent definition and the introduction of distributed simulations of Agent-Based Systems.

\subsection{Agent Definition}

Actually, there is no consensus in the scientific literature on the definition of an agent. Disciplines, in which reference is made, are numerous and various authors have proposed different definitions as for example Ferber [3]. However, the definition proposed in Jennings et al. [4] is commonly used within the MAS community: "an agent is a computer system, situated in some environment that is capable of flexible autonomous action in order to meet its design objectives...».

Agents and MAS constitute an active research field in which numerous applications are developed. In Nwana [5], the authors propose surveys of the agents according to various application domains (cognitive agents, software agents, mobile agents, etc.). Agents perceive the modifications of their environment and perform actions on it. Among the possible actions, agents have to determine the most suitable decisions that can reach their objectives. In addition to the application domain, environment, interaction and organization influence the design of the agent. 


\section{Perspectives to Interoperability}

From the state-of-the-art of federated enterprise interoperability and implementations experiences presented in $\S 2$, we can define several directions for, almost natural, interoperability barrier removal with Agent and HLA concepts in the following domains.

The first direction concerns the definition of commonly recognized paradigms and data structure able to evolve during run time. The second not addressed requirement at the enterprise modeling level is the data synchronization. The data exchanged order is crucial; ignoring this can lead to not desired indeterminist model behavior.

Finally, the enterprise modeling must consider the confidentiality management of data. The interoperability can be considered between concurrent enterprises in that context, a strategy of data sharing/not sharing between these must be defined. We present, in the following, propositions to address these requirements.

\section{Federated Enterprise Agents}

In the federated Enterprise Interoperability approach, no common persistent ontology is supposed to exist; the communication must be accommodated on the fly. In consequence, the ontology that structures the messages exchanged must be short-lived, (i.e. non-persistent). We state that the communication mechanism, in this approach, can be informally illustrated as follow in figure 1. We mainly distinguish two cases.

In case a., the enterprise 1 sends information and the ontology to decode it at the same time. This ontology is supposed to be only valid for this information.

In case b., the enterprise 1 sends only the information to enterprise 2 . Once enterprise 2 receives the information, it checks within its local ontology if it is able to decode the information. If not, it asks for the ontology associated to the message to the sender of the message. The new received ontology can be conserved to be reused with further data sent by the same emitter.

a.

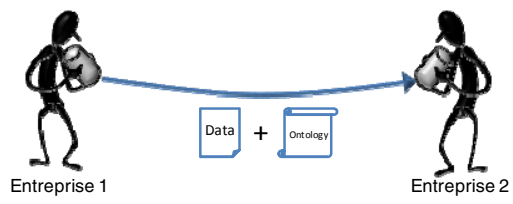

b.

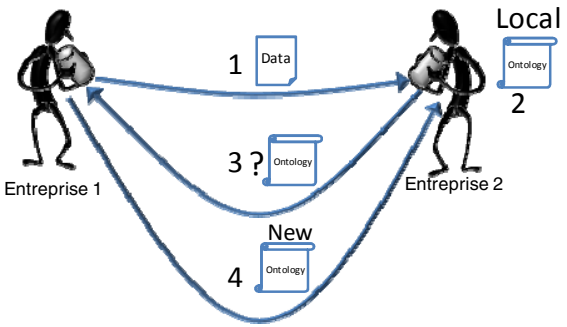

Fig. 1. HLA Federated Interoperability Data Exchange

In the first solution, the information size exchanged is more important, it can be intercepted and the confidentiality can be broken. In the second case, the confidentiality is enforced but it can require more exchanges between the two partners and consequently overlapping the communication duration. Nevertheless, for confidentiality 
(i.e. §5.) and accuracy to the definition of $\$ 2$., we choose to focus in this article, on the second solution. From that postulate, we introduce the concept of "short lived" ontology, where ontology can be, in some case, suppressed after use or have finite duration validity. It maps on-the-fly accommodation requirement of federated interoperability.

\subsection{Agent for Short-Lived Ontology Concept}

From the concept presented in the preceding point, we state that the autonomous dialogue between Agents, from Multi Agent System (MAS) [3] and Agent Based Simulation [6], can map properly the "on the fly" concept of federated interoperability at process level. We propose to use the dialogue mechanism algorithm of Agent programming, introduced in [3] and [6], to solve at computerized level the problem of federated Enterprise Interoperability. This dialogue between Agents, aims at establishing communication (e.g. two enterprises that discuss to agree on domain ontology), it is based on Agents cooperation behavior settings, and messages exchanged language (ontology).

On the one hand, (Ferber in [3] specifies the communication behavior algorithms of Agents' with Petri Nets (PN). On the other hand, Zacharewicz in [7], have tailored the use of DEVS/HLA (introduced as a generalized M\&S language (including PN) for distributed systems, gaining accuracy and flexibility (these models communicate within a distributed environment by message passing). Thus, we state that DEVS/HLA synchronized communication can support a unified, reusable and interoperating implementation of distributed Agents' dialogue.

On behalf of previous paragraphs propositions, we propose to develop a MAS simulator in the aim of validating Enterprise Interoperability concepts, studying the performance by simulation and implementing a concrete solution for Enterprise Interoperability ISs.

The research for developing MAS distributed Platforms is wide (as can denote for e.g. a repository of Agent-Based Simulation Platforms proposed in [8]), and actual MAS simulators are powerful (i.e. [6]). Nevertheless, they mainly do not tackle the problem of interoperability and reuse of components at coding level (e.g. heterogeneity of syntax, semantic, time management, etc.). In consequence, to preserve Interoperability at all levels of Enterprise Modeling including execution level; we propose to implement an Enterprises Federated Agents System that will be HLA compliant (to guaranty also run time interoperability between heterogeneous software components).

\section{Federated Communication Framework}

The proposition starts from the statement on interoperability needs on interfacing enterprises IS in the context of project cooperation. The figure 2 depicts the requirement on exchanging data from heterogeneous Information Systems, it can include vendor tools such as SAP and other specific developed solutions. It is issued by generalizing study case of [9], various enterprises are involved in a common project and their heterogeneous components need to be interfaced. Existing interoperability between components is represented with plain arrows and in demand interoperability 
with dotted arrows. Labarthe et al. in [10] reports on solution to establish interoperability using MAS in the communication of enterprises IS (i.e. figure 2 long dotted set); they have implemented an agent communication mechanism using JADE platform facilities. Zacharewicz et al. in [9] defined an interoperable Workflow using DEVS / HLA. By joining these approaches, this paper introduces the basis for a generalized approach to realize interoperability between heterogeneous components. A way of research is envisaged. The first requirement is solving the interoperability of data and services, HLA can be part of a solution.

For instance in the practical case of figure 2, a solution is to establish links to an "Interoperability Service Bus", referring to Enterprise Service Bus of Chapel [11] concepts, to connect new features with already connected components, (e.g. DEVS/HLA Anylogic, HLA...). We detail next point how an HLA compliant platform can facilitate the integration of all required components.

Different levels of ontology are required in our approach. From low level with poor semantics associated to HLA objects to information transport level (HLA bus). Agent KQML [12] will be used as an intermediate level able to match from low level description to high level description used in heterogeneous platforms, software or enterprise models involved in the system using reference to domain Ontologies.

Information System Services Layer. The distributed implementation requires the extension of two add-ons to the local enterprise IS to define HLA Enterprise Federate Agent (HEFA). We present in the figure 2, the elements of this new architecture. The respective local enterprise ISs remain unchanged, HLA only required to add components to interface with input output messages of the IS.

Multi Agent Coding / Decoding Layer. All agents involved in the data ontology matching are detailed:

The Agent 1 (Storing data) is employed to store the received information and will check the capacity to decode information using a communication with ontology agent. Receiving the agreement to use the data, it sends to the information system the data and the ontology. The Agent 2 (Ontology) is linked to a repository of local ontology; it checks the consistency of the information regarding the local ontology and decides if the data can be exploited. If yes, it sends back to the storing data agent the information and the ontology to use. If not, it asks to the communication agent to start dialog to obtain the appropriate ontology. The Agent 3 (Communication) will start a conversation with the respective agent of the data sender to deal on the modality to receive the appropriate ontology. We propose, in the following, that this dialog will be established using HLA message communication protocol but from a conceptual consideration it can be considered as a general approach where HLA is just one practical technical solution.

Local RTI Component Layer. This level is the lower level; it is the service and data level. It deals with technologies employed to exchange computerized information. The first component is the Local RTI component (LRC). We illustrate in figure 2, the use of two instances of our structure connected to a Workflow monitoring tool. This tool is employed to run a simulation of a Supply chain or of a document, exchanging process and is triggering right in time the information systems of the interoperating enterprises. 


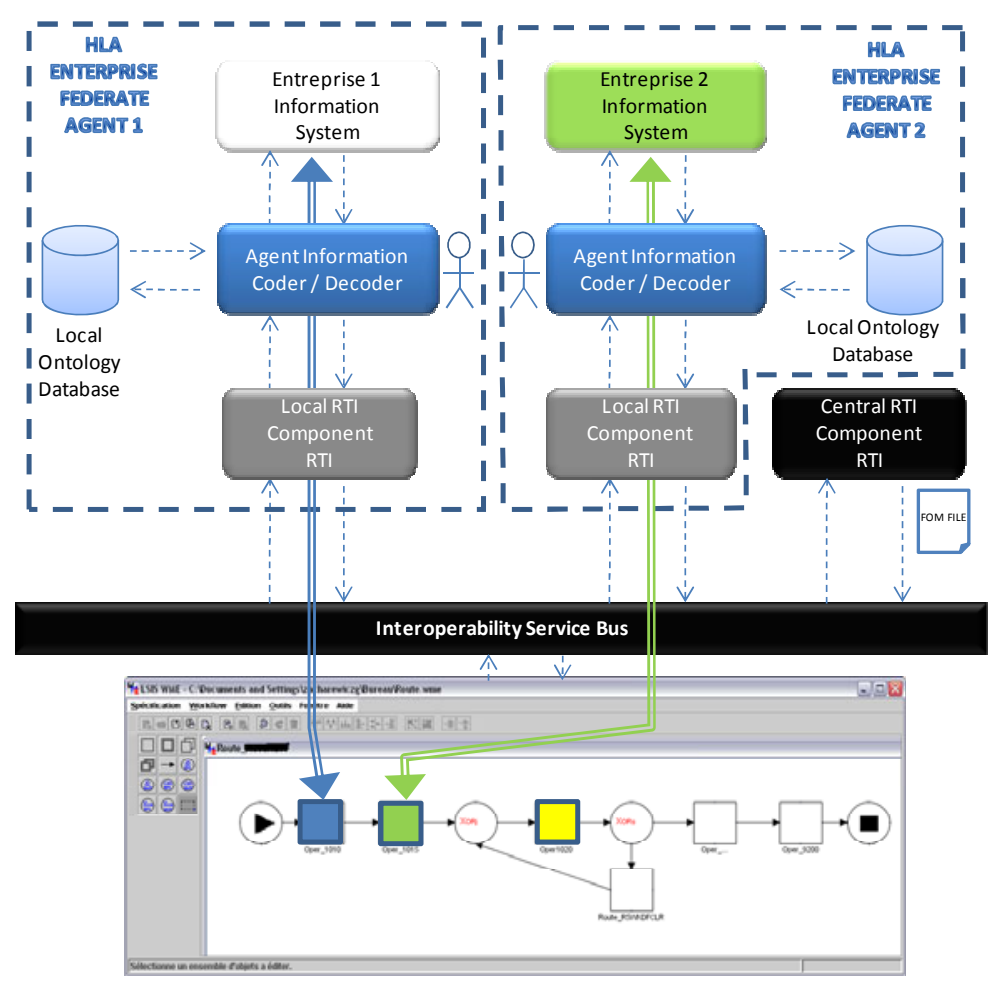

Fig. 2. Federated Interoperability Data Exchange

\subsection{HLA Interaction and Object Class Model}

The idea is to propose a new mapping for data to be exchanged in a HLA compliant distributed system between Interoperable Enterprise Agents. We proposed in [9], a generic HLA FOM that will support the descriptions of the data required to insure the exchange of information in the figure 1. case $b$.

Information to exchange. Information exchanged between enterprises information systems will be mapped with HLA Object class models (that handle persistent information in the distributed execution). The enterprise IS federates will publish and subscribe (PS) to these classes of information. An information channel Object represents the informational link between at least two enterprises. We notice that communication channels preserve confidentiality.

Ontology. Ontologies exchanged between enterprises are not persistent in the studied approach; they will be mapped into HLA Interactions (that are non persistent information exchanged). Enterprise IS Federate Agents will publish and subscribe (PS) to these classes of information. One Ontology class is associated to each information channel; each Ontology definition can change during run time. Eventually, validity duration can be set for each Ontology.

The structure of the generic ontology and of the messages will be implemented in the FOM presented in [9]. Because of generic concepts introduced in this paper, HLA 
interaction parameters and HLA object attributes are not fully specified; they will be in more detailed depending on the applications. It gives flexibility to the data structures exchanged.

\section{Conclusion and Future Work}

This article has given a state of the art of Enterprise Interoperability concepts and illustrated the use of Agent concepts and HLA standard for the implementation of enterprise applications federations.

From the new concept of short-lived enterprise ontology for federated Enterprise Interoperability, we proposed a specific implementation of distributed enterprise models for simulation or real time information exchange. At the end, the keys for implementation given by Agent dialogue mechanism has helped to bridge the gap from Enterprise Interoperability concepts to HLA compliant distributed implementation in the field of Enterprise Modeling by following a new standardized and systematic approach.

\section{References}

1. INTEROP: INTEROP Home Page, INTEROP NoE (2005), http: / / www. interop-vlab.eu /

2. IEEE std 1516.22000: IEEE Standard for Modeling and Simulation (M\&S) High Level Architecture (HLA) - Federate Interface Specification. IEEE, New York (2000)

3. Ferber, J.: Multi-agents Systems. In: An Introduction to Distributed Artificial Intelligence. Addison Wesley, London (1999)

4. Jennings, N.R., Sycara, K., Wooldridge, M.: A Roadmap of Agent Research and Development. Autonomous Agents and Multi-agents Systems 1(1), 7-38 (1998)

5. Nwana, H.S.: Software agents: An overview. Knowledge Engineering Review 11(3), 205244 (1996)

6. Huang, C.Y., Nof, S.Y.: Autonomy and viability measures for Agent-based manufacturing systems. International Journal of Production Research 38(17), 4129-4148 (2000)

7. Zacharewicz, G., Frydman, C., Giambiasi, N.: G-DEVS/HLA Environment for Distributed Simulations of Workflows. Simulation 84(5), 197-213 (2008)

8. Marietto, M.B., David, N., Sichman, J.S., Coelho, H.: Requirements Analysis and AgentBased Simulation Platforms: State of the Art and New Prospects. In: Sichman, J.S., Bousquet, F., Davidsson, P. (eds.) MABS 2002. LNCS (LNAI), vol. 2581, pp. 125-141. Springer, Heidelberg (2003)

9. Zacharewicz, G., Chen, D., Vallespir, B.: Short-Lived Ontology Approach for Agent/HLA Federated Enterprise Interoperability. In: IEEE Proc. of International Conference I-ESA Interoperability for Enterprise Software and Applications, Beijing (2009)

10. Labarthe, O., Espinasse, B., Ferrarini, A., Montreuil, B.: Toward a Methodological Framework for Agent-Based Modelling and Simulation of Supply Chains in a Mass Customization Context. Simulation Modelling Practice and Theory 15(2), 113-136 (2007)

11. Chappell, D.A.: Enterprise Service Bus. O’Reilly, Sebastopol (2004)

12. Finin, T., Fritzson, R., McKay, D., McEntire, R.: KQML as an agent communication language. In: Proc. of the Third International Conference on Information and Knowledge Management, Gaithersburg, Maryland, USA (1994) 